\title{
Collectivity and Periodic Orbits in a Chain of Interacting, Kicked Spins
}

\author{
M. Akila, D. Waltner, B. Gutkin, P. Braun and T. Guhr \\ Fakultät für Physik, Universität Duisburg-Essen, Lotharstr. 1, 47048 Duisburg, Germany
}

\begin{abstract}
The field of quantum chaos originated in the study of spectral statistics for interacting many-body systems, but this heritage was almost forgotten when single-particle systems moved into the focus. In recent years new interest emerged in many-body aspects of quantum chaos. We study a chain of interacting, kicked spins and carry out a semiclassical analysis that is capable of identifying all kinds of genuine many-body periodic orbits. We show that the collective many-body periodic orbits can fully dominate the spectra in certain cases.
\end{abstract}

DOI: 10.12693/APhysPolA.132.1661

PACS/topics: quantum chaos, many-body systems, semiclassics

\section{Introduction}

Random matrix theory (RMT) was developed and used starting in the 50's to study statistical aspects of nuclei and other interacting many-body systems, see Refs. [1-3]. Later on, it was realized that RMT also works for single-particle systems [4-7], prompting the celebrated Bohigas-Giannoni-Schmit (BGS) conjecture. Semiclassical analysis revealed that the classical periodic orbits (POs) are the skeleton of the quantum spectrum $[1,2,8-11]$ also providing strong support for the BGS conjecture [12-14]. It was almost forgotten that many-body systems were the objects of interest in early quantum chaos. Only recently, new attempts to address many-body systems in the present context were put forward, e.g. many-body localization [17-19] also observed in recent experiments [20, 21], spreading in self-bound many-body systems [22, 23], a semiclassical analysis of correlated many-particle paths in the BoseHubbard chains [24] and a trace formula connecting the energy levels to the classical many-body orbits [25, 26]. There are also attempts to study field theories semiclassically [27]. As two large parameters exist in many-body systems, the number of particles $N$ and the Hilbert space dimension determined by the inverse effective Planck constant $\hbar_{\text {eff }}^{-1}$, different semiclassical limits are meaningful [28].

Many-body systems show collective motion, not present in single-particle systems. By collectivity we mean a coherent motion of all or of large groups of particles which can be identified in the classical phase space as well as in the quantum dynamics. Typically, a manybody system exhibits incoherent, i.e. non-collective, motion of its particles, coherent collective motion and forms of motion in between. Collectivity has a strong impact on the level statistics. While incoherent particle motion leads to RMT statistics as in the famous example of the nuclear data ensemble $[29,30]$, collective excitations often show the Poisson statistics typical for integrable systems, as e.g. in Ref. [31], see Ref. [3]. Due to the mixed phase space, the BGS conjecture is not directly applicable to many-body systems.
To illuminate the full complexity of the motion in many-body systems and the importance of collectivity from a semiclassical viewpoint, we consider a chain of $N$ interacting kicked spins. We focus on the short time regime but consider arbitrary $N$, where the collectivity plays a significant role. Thereby, we provide a better understanding of spin chain dynamics as this class of systems is presently in the focus of theoretical [32-35] and experimental [36-39] research. This presentation is based on our recent Letter [40].

\section{Chain of interacting, kicked spins}

Consider $N$ kicked spins with nearest neighbor interaction as in Ref. [41], described by the Hamiltonian

$$
\hat{H}=\hat{H}_{I}+\hat{H}_{K} \sum_{T=-\infty}^{\infty} \delta(t-T)
$$

with the interaction part $\hat{H}_{I}$ and the kick part $\hat{H}_{K}$,

$$
\hat{H}_{I}=\sum_{n=1}^{N} \frac{4 J \hat{s}_{n+1}^{z} \hat{s}_{n}^{z}}{(j+1 / 2)^{2}}, \quad \hat{H}_{K}=\frac{2}{j+1 / 2} \sum_{n=1}^{N} \boldsymbol{b} \cdot \hat{\boldsymbol{s}}_{n}
$$

where $\hat{s}_{n}=\left(\hat{s}_{n}^{x}, \hat{s}_{n}^{y}, \hat{s}_{n}^{z}\right)$ are the operators for spin $n$ and quantum number $j$. Periodic boundary conditions, i.e. $\hat{s}_{N+1}^{z}=\hat{s}_{1}^{z}$, make the system translation invariant. Moreover, $J$ is the coupling constant and $b$ a magnetic field, assumed without loss of generality to have the form $\boldsymbol{b}=\left(b^{x}, 0, b^{z}\right)$. The kicks act at discrete integer times $T$. The one period time-evolution (Floquet) operator reads

$$
\begin{aligned}
& \hat{U}=\hat{U}_{I} \hat{U}_{K}, \quad \hat{U}_{I}=\mathrm{e}^{-\mathrm{i}(j+1 / 2) \hat{H}_{I}}, \\
& \hat{U}_{K}=\mathrm{e}^{-\mathrm{i}(j+1 / 2) \hat{H}_{K}},
\end{aligned}
$$

where $(j+1 / 2)^{-1}$ takes on the role of the Planck constant $\hbar_{\text {eff }}$. We find the corresponding classical system by replacing $\hat{\boldsymbol{s}}_{m} \rightarrow \sqrt{j(j+1)} \boldsymbol{n}_{m}$ with a classical spin unit vector $\boldsymbol{n}_{m}$ precessing on the Bloch sphere. The time evolution can therefore be interpreted as the action of two subsequent rotation matrices

$$
\boldsymbol{n}_{m}(T+1)=R_{\boldsymbol{z}}\left(4 J \chi_{m}\right) R_{\boldsymbol{b}}(2|\boldsymbol{b}|) \boldsymbol{n}_{m}(T),
$$

first around the magnetic field axis and then around 
the $z$ axis (the Ising part) with angle $4 J \chi_{m}, \chi_{m}=$ $n_{m-1}^{z}+n_{m+1}^{z}$. The classical system can be cast in Hamiltonian form,

$$
\begin{aligned}
& H(\boldsymbol{q}, \boldsymbol{p})=\sum_{n=1}^{N}\left[4 J p_{n+1} p_{n}\right. \\
& \left.+\sum_{T=-\infty}^{\infty} \delta(t-T) 2\left(b^{z} p_{n}+b^{x} \sqrt{1-p_{n}^{2}} \cos q_{n}\right)\right],
\end{aligned}
$$

from which the canonical equations follow. The $N$ component vectors $\boldsymbol{p}$ and $\boldsymbol{q}$ are the conjugate momenta and positions of the $N$ (classical) spins, respectively. The vectors on the Bloch sphere are given by

$$
\boldsymbol{n}_{m}=\left(\sqrt{1-p_{m}^{2}} \cos q_{m}, \sqrt{1-p_{m}^{2}} \sin q_{m}, p_{m}\right)
$$

in terms of the canonical variables. In our study, the magnetic field $\boldsymbol{b}$ has a sizeable angle with the $z$ axis to ensure non-trivial chaotic motion.

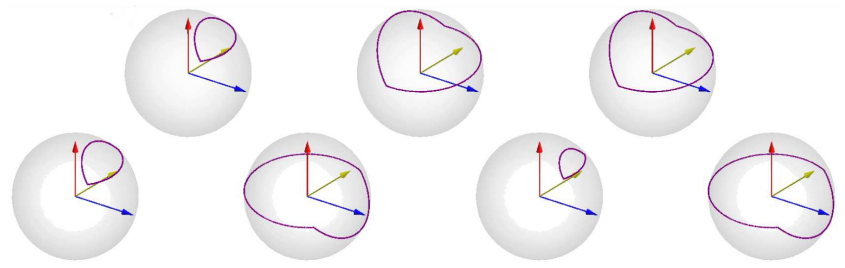

Fig. 1. Example for the classical motion of $N=7$ spins. Periodic orbits for $T=1$ kick.

An example for the classical periodic orbits is shown in Fig. 1 in the case of $N=7$ spins and $T=1$ kick.

\section{Semiclassics and periodic orbits}

In Ref. [42] we recently expressed the trace of the propagator $\hat{U}$ to power $T$ for an interacting spin system in a Gutzwiller-type-of form valid in the limit $j \rightarrow \infty$,

$$
\operatorname{Tr} \hat{U}^{T} \sim \sum_{\gamma(T)} A_{\gamma} \mathrm{e}^{\mathrm{i}(j+1 / 2) S_{\gamma}} .
$$

This is a sum over classical periodic orbits (POs) $\gamma$ of duration $T$ if they are well isolated. Here, $S_{\gamma}$ is the classical action and, for an isolated orbit, $A_{\gamma}$ the stability amplitude. For the Hamiltonian (5), most POs are neither fully stable nor unstable. The connection between the classical and the quantum system is revealed by the Fourier transform $\rho(S)$ of Eq. (7) in $j$. This is methodically similar to Refs. [10,11] and was also used for the kicked top [43, 44]. We find

$$
\begin{aligned}
& \rho(S)=\frac{1}{j_{\text {cut }}} \sum_{j=1}^{j_{\text {cut }}} \mathrm{e}^{-\mathrm{i}(j+1 / 2) S} \operatorname{Tr} \hat{U}^{T} \stackrel{j_{\text {cut }} \rightarrow \infty}{\sim} \\
& \frac{1}{j_{\text {cut }}} \sum_{\gamma(T)} A_{\gamma} \delta\left(S-S_{\gamma}\right),
\end{aligned}
$$

which approximates the action spectrum by peaks of width approximately $\pi / j_{\text {cut }}$ whose positions are given by the actions modulo $2 \pi$ of the POs with length $T$.

\section{Explosion of dimension and duality relation}

At this point, we have to overcome a severe problem. To resolve the peaks in $\rho(S)$ we need to compute $\operatorname{Tr} \hat{U}^{T}$ for sufficiently large $j_{\text {cut }}$. But as its matrix dimension $(2 j+1)^{N} \times(2 j+1)^{N}$ grows exponentially with $N$, a direct calculation of the spectrum of $\hat{U}$ is impossible, e.g., even the propagator $\hat{U}^{T}$ for $N=19$ spins at $j=1$ has a matrix dimension of $10^{9} \times 10^{9}$. Luckily, recently developed duality relations $[28,45]$ provide the solution and make possible, for the first time, a semiclassical analysis of genuine many-body orbits. The crucial ingredient is the exact identity

$$
\operatorname{Tr} \hat{U}^{T}=\operatorname{Tr} \hat{W}^{N}
$$

The trace over the time-evolution operator $\hat{U}$ for $T$ time steps equals the trace over a nonunitary "particlenumber-evolution" operator $\hat{W}$ for $N$ particles. Its dimension $(2 j+1)^{T} \times(2 j+1)^{T}$ is governed by $T$ instead of $N$. A cartoon-type-of visualization of the duality relation is given in Fig. 2.

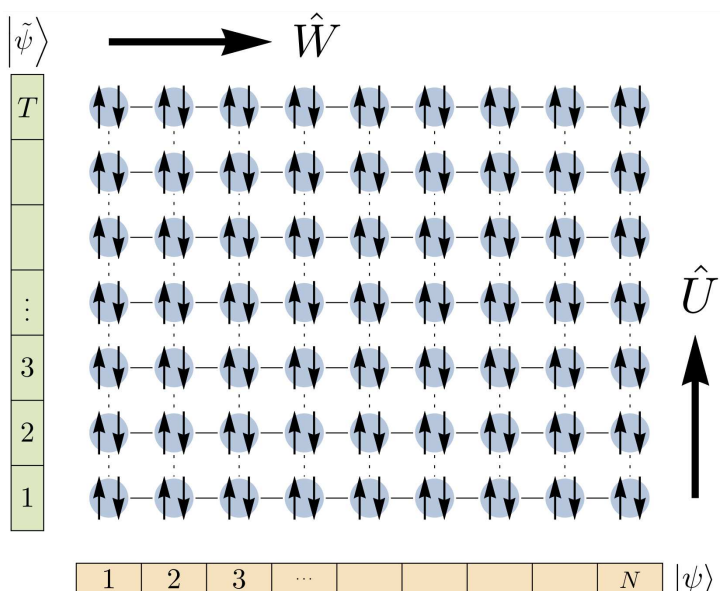

Fig. 2. Attempt to visualize the duality relation (8).

This duality allows us to calculate $\rho(S)$ for arbitrary $N$ as long as $T$ is sufficiently short. In Refs. [40, 46] we generalize this duality approach, developed for $j=1 / 2$ in Ref. [45], to $j \gg 1$. The dual "particle-number-evolution" operator is a product as well, $\hat{W}=\hat{W}_{I} \hat{W}_{K}$. We give its explicit form using a $(2 j+1)^{T}$ dimensional product basis in spin space,

$$
|\boldsymbol{\sigma}\rangle=\left|\sigma_{1}\right\rangle \otimes\left|\sigma_{2}\right\rangle \otimes \cdots \otimes\left|\sigma_{T}\right\rangle
$$

with discrete single spin states $\sigma_{t} \in\{-j,-j+1, \ldots+j\}$. The interaction part is diagonal with matrix elements

$$
\left\langle\boldsymbol{\sigma}\left|\hat{W}_{I}\right| \boldsymbol{\sigma}^{\prime}\right\rangle=\delta_{\boldsymbol{\sigma}, \boldsymbol{\sigma}^{\prime}} \prod_{t=1}^{\dagger}\left\langle\sigma_{t}\left|\exp \frac{2 \mathrm{i} \boldsymbol{b} \cdot \hat{\boldsymbol{s}}}{j+1 / 2}\right| \sigma_{t+1}\right\rangle .
$$

The boundary conditions are periodic, i.e. $T+1=1$. The kick part, however, must have a local structure

$$
\hat{W}_{K}=\otimes_{t=1}^{\dagger} \hat{w}_{K}, \quad\left\langle\sigma\left|\hat{w}_{K}\right| \sigma^{\prime}\right\rangle=\exp \frac{4 \mathrm{i} J \sigma \sigma^{\prime}}{(j+1 / 2)^{2}} .
$$

Although $\hat{w}_{K}$ is related to the interaction of $\hat{U}_{I}$ it is not 
diagonal. In the integrable case $\left(b^{x}=0\right)$ the dual operator acquires particularly simple form which we give for illustrative purposes,

$$
\begin{aligned}
& \hat{W}_{n m}=\exp g\left(\mathrm{i} \frac{4 J T}{j+1 / 2}(n-j-1)(m-j-1)\right. \\
& \left.\quad+2 \mathrm{i} T b^{z}(n-j-1) g\right) .
\end{aligned}
$$

The indices $m, n$ run from 1 to $2 j+1$ and time turns, in this case only, to a value set by the system parameters. For further details, see Refs. [40, 46].

\section{Dominance of collectivity in classical action spectra}

We numerically calculate action spectra $|\rho(S)|$ for $T=$ 1 and $T=2$ kicks, thereby exploring the short-time behavior. We do this by, first, evaluating the traces of the quantum mechanical time-evolution operator with the duality relation and, second, by computing the classical periodic orbits. Hence, we obtain the action spectra in both ways indicated in Eq. (8). We begin with $N=19$ spins and $T=1$ kick, both calculations are shown in
Fig. 3. The positions of the periodic orbits are indicated below the horizontal line at zero. Very good agreement is seen even for the peak heights. We now turn to $T=2$ kicks.

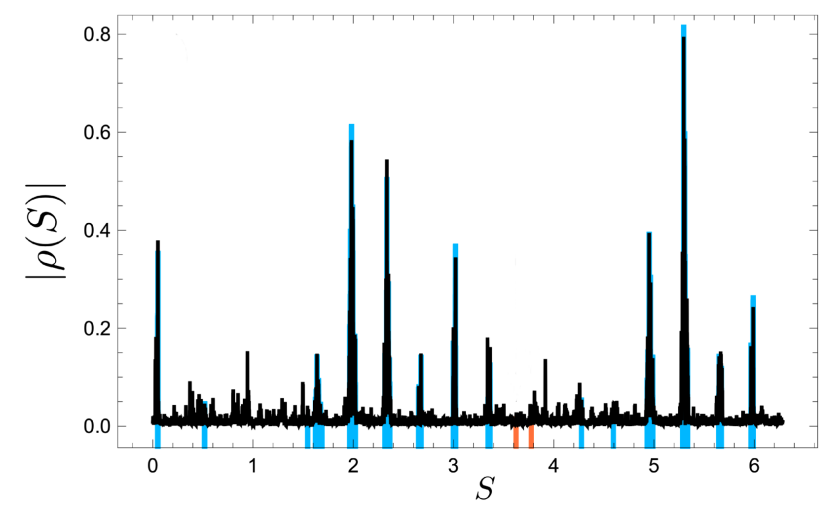

Fig. 3. Action spectrum for $N=19$ spins and $T=1$ kick.
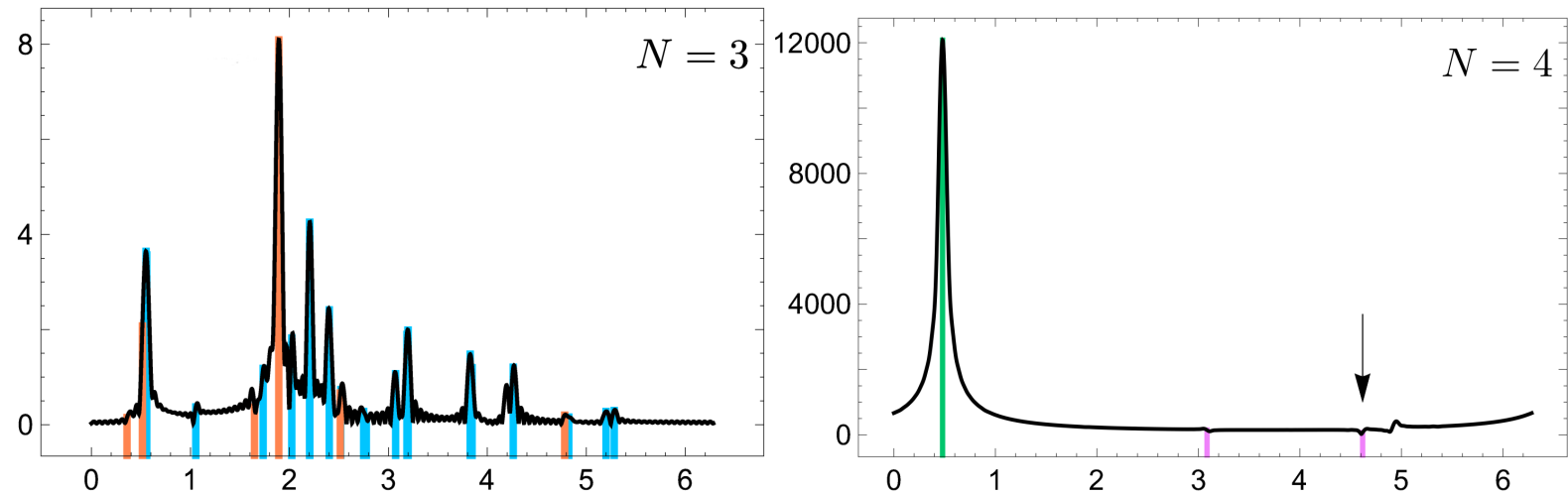

Fig. 4. Action spectra for $N=3$ and $N=4$ spins on the left and right hand side, respectively, for $T=2$ kicks.
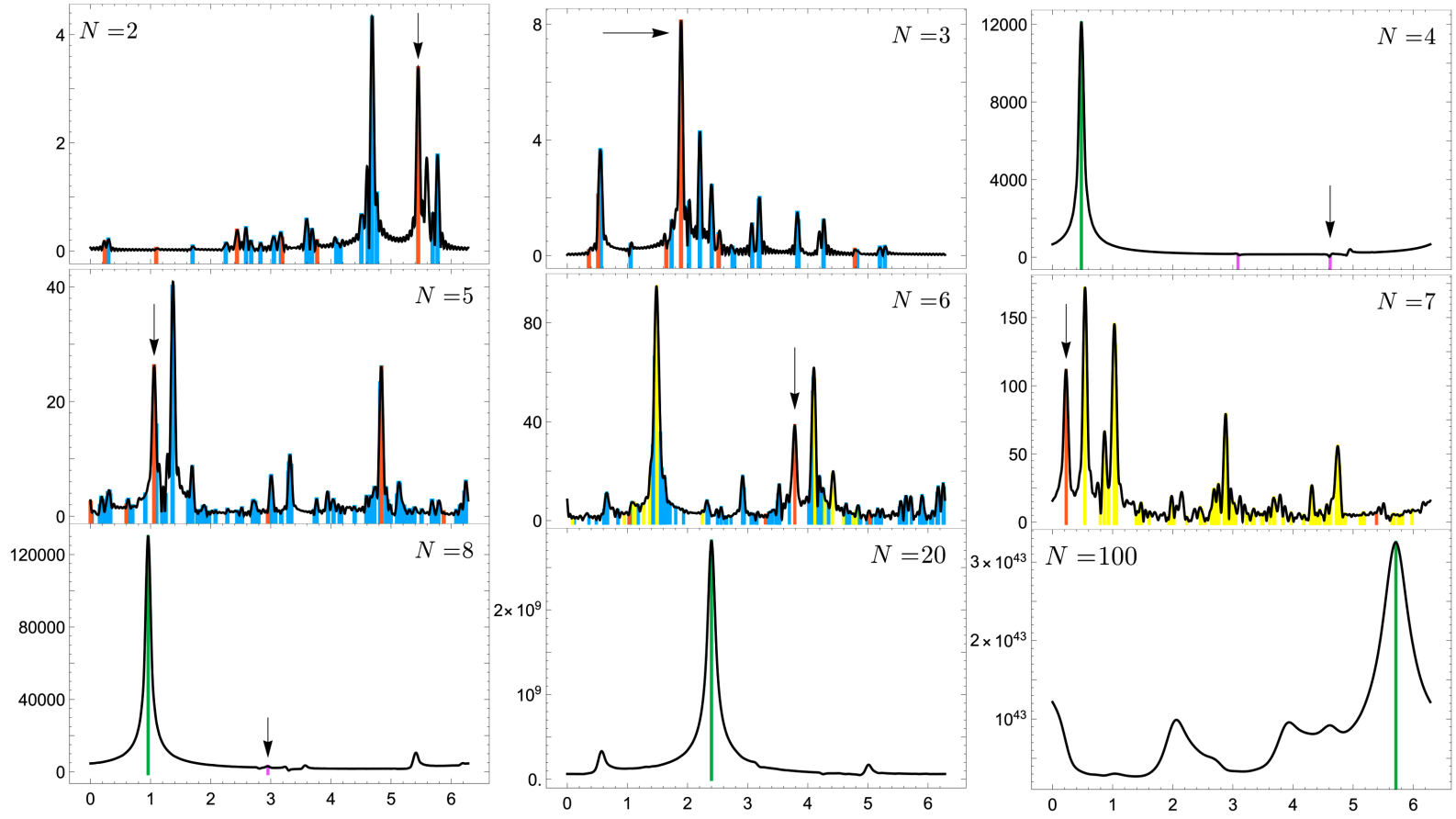

Fig. 5. Action spectra for $N=2,3,4,5,6,7,8,20,100$ spins for $T=2$ kicks. 
As depicted in Fig. 4, the action spectra differ strongly for $N=3$ and $N=4$ spins. We are led to argue that, in the case $T=2$, the motion for $N=3$ spins is largely incoherent motion of the spins, while it is coherent and collective for $N=4$ spins. This can be understood by looking, always in the case $T=2$, at the action spectra for a varying numbers of spins in Fig. 5. Whenever the number $N$ of spins is an integer multiple of four, the spectra are dominated by one very large peak which is much higher than in the case of the other numbers $N$ of spins. Careful analysis of the classical phase space yields an explanation by revealing the occurrence of four-dimensional manifolds of non-isolated periodic orbits with equal actions. The effect is illustrated in Fig. 6. Whenever the number $N$

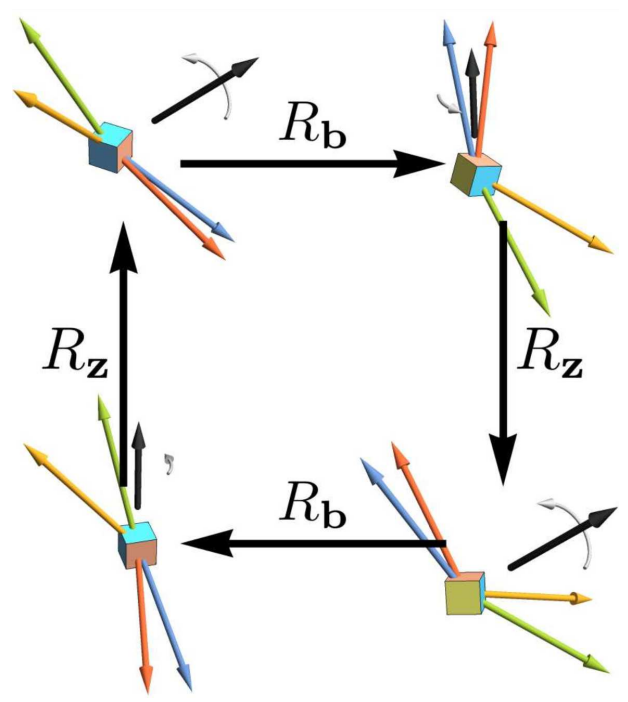

Fig. 6. Rigid-body-type-of rotation of all groups af four spins if the number of spins $N$ is an integer mutiple of four.

of spins is an integer multiple of four, the spins organize themselves into subgroups of four spins each which perform a rigid-body-type-of rotation in which these four spins do not exhibit any kind of relative motion. This is a strongly coherent, collective motion which, as Fig. 5 shows, outpowers the individual incoherent motion, completely dominating the action spectra. This phenomenon cannot be isolated. We expect similar, yet geometrically different, forms of collective motion for other numbers $N$ of spins and other numbers $T$ of kicks.

\section{Conclusions}

We carried out a semiclassical analysis of a (nonintegrable) interacting, many-body quantum system. We studied a kicked spin chain representing a class of systems presently being in the focus of experimental and theoretical research. For the first time, we presented a unifying semiclassical approach to incoherent and to coherent, collective dynamics. Such an interplay between different kinds of motion is common to very many, if not all, large systems. The key tool was a recently discovered duality relation between the evolutions in time and particle number. It outmaneuvers the drastically increasing complexity of the problem with growing particle number. In the spin chain a certain type of collective motion strongly dominates the spectra, whenever the particle number is an integer multiple of four. An experimental verification is likely to be feasible in view of the improving ability to control systems with larger numbers of spins.

\section{Acknowledgments}

One of us (T.G.) is grateful to the organizers of the 8th Workshop on Quantum Chaos and Localisation Phenomena in Warsaw, Poland, May 2017, where this research was presented.

\section{References}

[1] F. Haake, Quantum Signatures of Chaos, Springer, 2010.

[2] H.-J. Stöckmann, Quantum Chaos - An Introduction, Cambridge University Press, 1999.

[3] T. Guhr, A. Müller-Groeling, H.A. Weidenmüller, Phys. Rep. 299, 189 (1998).

[4] S.W. McDonald, A.N. Kaufman, Phys. Rev. Lett. 42, 1189 (1979).

[5] G. Casati, F. Valz-Gris, I. Guarneri, Lett. Nuovo Cim. 28, 279 (1980).

[6] M.V. Berry, Ann. Phys. (New York) 131, 163 (1981).

[7] O. Bohigas, M.J. Giannoni, C. Schmit, Phys. Rev. Lett. 52, 1 (1984).

[8] P. Cvitanović, R. Artuso, P. Dahlqvist, R. Mainieri, G. Tanner, G. Vattay, Chaos: Classical and Quantum, ChaosBook.org, Niels Bohr Institute, Copenhagen 2016.

[9] D. Wintgen, H. Friedrich, Phys. Rev. Lett. 57, 571 (1986).

[10] A. Holle, J. Main, G. Wiebusch, H. Rottke, K.H. Welge, Phys. Rev. Lett. 61, 161 (1988).

[11] D. Wintgen, Phys. Rev. Lett. 58, 1589 (1987).

[12] M.V. Berry, Proc. R. Soc. Lond. A 400, 229 (1985).

[13] M. Sieber, K. Richter, Phys. Scr. T, 90, 128 (2001).

[14] S. Heusler, S. Müller, A. Altland, P. Braun, F. Haake, Phys. Rev. Lett. 98, 044103 (2007).

[15] F. Wegner, Phys. Rep. 67, 15 (1980).

[16] K.B. Efetov, Adv. Phys. 32, 53 (1983).

[17] B.L. Altshuler, Y. Gefen, A. Kamenev, L.S. Levitov, Phys. Rev. Lett. 78, 2803 (1997).

[18] B.M. Basko, I.L. Aleiner, B.L. Altshuler, Ann. Phys. 321, 1126 (2006).

[19] M. Žnidarič, T. Prosen, P. Prelovšek, Phys. Rev. B 77, 064426 (2008).

[20] M. Schreiber, S.S. Hodgman, P. Bordia, H.P. Lüschen, M.H. Fischer, R. Vosk, E. Altman, U. Schneider, I. Bloch, Science 349, 842 (2015).

[21] J.-Y. Choi, S. Hild, J. Zeiher, P. Schauß, A. RubioAbadal, T. Yefsah, V. Khemani, D.A. Huse, I. Bloch, C. Gross, Science 352, 1547 (2016).

[22] J. Hämmerling, B. Gutkin, T. Guhr, J. Phys. A 43 , 265101 (2010);, Europhys. Lett. 96, 20007 (2011). 
[23] J. Freese, B. Gutkin, T. Guhr, Physica A 461, 683 (2016).

[24] T. Engl, J. Dujardin, A. Argüelles, P. Schlagheck, K. Richter, J.D. Urbina, Phys. Rev. Lett. 112 , 140403 (2014).

[25] T. Engl, J.D. Urbina, K. Richter, Phys. Rev. E 92, 062907 (2015).

[26] R. Dubertrand, S. Müller, New J. Phys. 18, 033009 (2016).

[27] P. Cvitanović, Physica A 288, 61 (2000).

[28] B. Gutkin, V. Osipov, Nonlinearity 29, 325 (2016).

[29] R.U. Haq, A. Pandey, O. Bohigas, Phys. Rev. Lett. 48, 1086 (1982).

[30] O. Bohigas, R.U. Haq, A. Pandey, in: Nuclear Data for Science and Technology, Ed. K.H. Bockhoff, Reidel, Dordrecht 1983, p. 809.

[31] J. Enders, T. Guhr, N. Huxel, P. von Neumann-Cosel C. Rangacharyulu, A. Richter, Phys. Lett. B 486, 273 (2000).

[32] C. Braun, F. Li, A. Garg, M. Stone, J. Math. Phys. 56, 122106 (2015).

[33] M. Gessner, V.M. Bastidas, T. Brandes, A. Buchleitner, Phys. Rev. B 93, 155153 (2016).

[34] Y.Y. Atas, E. Bogomolny, J. Phys. A 47, 335201 (2014).
[35] J.P. Keating, N. Linden, H.J. Wells, Commun. Math. Phys. 338, 81 (2015).

[36] J. Simon, W.S. Bakr, R. Ma, M.E. Tai, P.M. Preiss, M. Greiner, Nature 472, 307 (2011).

[37] C. Neill, et al., Nat. Phys. 12, 1037 (2016).

[38] K. Kim, M.-S. Chang, S. Korenblit, R. Islam, E.E. Edwards, J.K. Freericks, G.-D. Lin, L.-M. Duan, C. Monroe, Nature 465, 590 (2010).

[39] J. Smith, A. Lee, P. Richerme, B. Neyenhuis, P.W. Hess, P. Hauke, M. Heyl, D.A. Huse, C. Monroe, Nat. Phys. 12, 907 (2016).

[40] M. Akila, D. Waltner, B. Gutkin, P. Braun, T. Guhr, Phys. Rev. Lett. 118, 164101 (2017).

[41] T. Prosen, Phys. Rev. E 65, 036208 (2002).

[42] D. Waltner, P. Braun, M. Akila, T. Guhr, J. Phys. A 50, 085304 (2017).

[43] H. Schomerus, M. Sieber, J. Phys. A 30, 4537 (1997).

[44] M. Kuś, F. Haake, D. Delande, Phys. Rev. Lett. 71, 2167 (1993).

[45] M. Akila, D. Waltner, B. Gutkin, T. Guhr, J. Phys. A 49, 375101 (2016).

[46] M. Akila, B. Gutkin, P. Braun, D. Waltner, T. Guhr, arXiv:1709.03601. 